\title{
Dynamics and Spectral Weights of Shake-up Valence Excitations in Transition Metal $K$-edge Resonant Inelastic X-ray Scattering
}

\author{
K. H. Ahn, ${ }^{1}$ A. J. Fedro, ${ }^{2,3}$ and Michel van Veenendaal ${ }^{2,3}$ \\ ${ }^{1}$ Department of Physics, New Jersey Institute of Technology, Newark, New Jersey, 07102 \\ ${ }^{2}$ Advanced Photon Source, Argonne National Laboratory, \\ 9700 South Cass Avenue, Argonne, Illinois 60439 \\ ${ }^{3}$ Department of Physics, Northern Illinois University, De Kalb, Illinois 60115
}

\begin{abstract}
Using a model Hamiltonian, we discuss how we could interpret data obtained from transition metal $K$-edge resonant inelastic x-ray scattering (RIXS) experiments. By analyzing the creation of valence excitations from the screening of the core hole and calculating corresponding RIXS spectra for metals and insulators, we find that the probability for excitations depends not only on the total energy but also on the asymmetric screening dynamics between electrons and holes.
\end{abstract}

PACS numbers: 78.70.Ck, 71.20.-b, 71.10.-w

\section{INTRODUCTION}

The inelastic x-ray scattering cross section from a solid state system is enhanced by orders of magnitude, when the incoming x-ray energy $\omega_{\text {in }}$ is tuned to a resonance of the system. For resonant inelastic x-ray scattering (RIXS), an electron is excited from a deep-lying core state into the valence shell. In particular, the RIXS at transition metal $K$-edges, which is the focus of this work, has drawn significant attention recently $1,2,3,4,5,6,7,8,9,10$ As illustrated in Fig. 1, transition metal $K$-edge RIXS involves a dipolar transition from the $1 s$ shell into the wide $4 p$ band [Fig. 1(a)], the screening of the $1 s$ core hole by the $3 d$ valence electrons [Fig. [1(b)], and the recombination of the $4 p$ electron and the $1 s$ core hole with the emission of a photon [Fig. [(c)]. Unlike RIXS at other edges (e.g. transition-metal $L$-edges), the dipole matrix elements in transition metal $K$-edge RIXS at a particular incoming photon energy $\left(\omega_{\text {in }}\right)$ and momentum $\left(\mathbf{q}_{\text {in }}\right)$ are constant factors, which makes transition metal $K$-edge RIXS a direct probe for the screening dynamics. The momentum (qout) and energy ( $\left.\omega_{\text {out }}\right)$ dependence of the outgoing x-ray resulting from the radiative decay of the core hole allows measurements of the energy $\omega=\omega_{\text {in }}-\omega_{\text {out }}$ and the momentum $\mathbf{q}=\mathbf{q}_{\text {in }}-\mathbf{q}_{\text {out }}$ of elementary excitations near the Fermi level or across the gap. The RIXS has several advantages compared to other spectroscopic probes. In contrast to angle-resolved photoemission, RIXS is bulk-sensitive due to the large penetration depth of x-rays. The use of a particular resonance makes RIXS chemically selective. As opposed to x-ray absorption, there is no core hole present in the final state. The RIXS has provided unique insights into, e.g., the momentum dispersion of charge excitations and magnetic excitations in high- $T_{c}$ superconductors and related systems, $2,3,4,4,5,6,7$ orbital excitations,,$\underline{8,9}$ and magnetic-ordering dependent transfer of spectral weight in colossal magnetoresistive manganites $\underline{10}$

Numerical calculations of the RIXS spectrum have

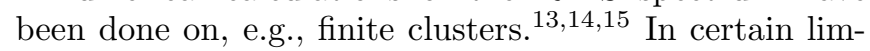
its, the RIXS cross section can be related to the dynamic (a)

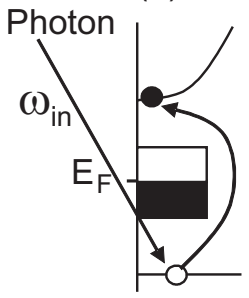

(b)

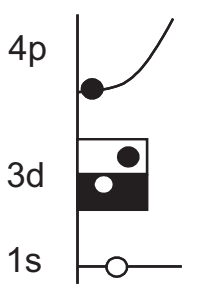

(c)

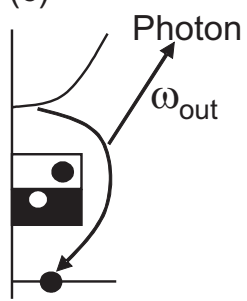

FIG. 1: Illustration of the RIXS process studied in our work, that is, transition metal oxide $K$-edge RIXS. This process is distinguished from other types of RIXS, such as transition metal oxide $L$-edge RIXS (Figures 1 and 2 in Ref. 11), or $K$-edge RIXS for diamond or graphite(Figure 3 in Ref. 12).

structure factor $S_{\mathbf{q}}(\omega)$, either by lowest order perturbation theory ${ }^{3.6}$ or by an approximate representation of the intermediate-state dynamics. 16 Within these approximations, the probability for the intermediate state core-hole potential to create excitations, such as electron-hole (e-h) pairs, mainly depends on the total energy of the shakeup process. In this work, we show that RIXS depends on the energies of the constituents of the excitation, leading to a strong asymmetry in electron and hole responses to the core hole for the parameter range where the screening dynamics become important. We demonstrate that the RIXS spectral line shape is determined by the nature of the intermediate-state core-hole valence-shell-electron excitons.

\section{MODEL HAMILTONIAN AND METHODS}

The total Hamiltonian for the RIXS process shown in Fig. 1 has electronic, photonic, and electron-photon interaction parts,

$$
H_{\text {total }}=H_{\mathrm{el}}+H_{\mathrm{pht}}+H_{\mathrm{el}-\mathrm{pht}} .
$$


The RIXS intensity is obtained from

$$
I\left(\omega_{\text {in }}, \omega_{\text {out }}\right)=\frac{2 \pi}{\hbar} \sum_{f}\left|U_{i \rightarrow f}\right|^{2} \delta\left(E_{f}+\omega_{\text {out }}-E_{i}-\omega_{\text {in }}\right),
$$

where $U_{i \rightarrow f}$ is the transition amplitude from the initial state $|i\rangle$ to a final state $|f\rangle$. In terms of the initial many-electron energy $E_{i}$, many-electron eigenstate $|n\rangle$ and eigenenergy $E_{n}$ of $H_{\mathrm{el}}$, and the FWHM intermediatestate lifetime-broadening $2 \Gamma$, the transition amplitude is given by

$$
U_{i \rightarrow f}=\sum_{n} \frac{\left\langle f\left|H_{\mathrm{el}-\mathrm{pht}}\right| n\right\rangle\left\langle n\left|H_{\mathrm{el}-\mathrm{pht}}\right| i\right\rangle}{E_{i}+\omega_{\mathrm{in}}-E_{n}+i \Gamma} .
$$

The Coulomb interaction between localized $1 s$ holes and $3 d$ electrons, which is the central mechanism for transition metal $K$-edge RIXS, is $U_{c}=4-7 \mathrm{eV}$ (Refs. 5, 17, 13, and 18). The excited $4 p$ electron is highly delocalized, and its Coulomb interaction with $1 s$ holes or $3 d$ electrons is neglected as an approximation, leading to

$$
H_{\mathrm{el}}=H_{\underline{1 s}}+H_{3 d}+H_{\underline{1 s}-3 d}+H_{4 p},
$$

where $H_{\underline{1 s}}, H_{3 d}$, and $H_{4 p}$ are Hamiltonians for $1 s, 3 d$ and $4 p$ levels, and $H_{1 s-3 d}$ represents $1 s-3 d$ Coulomb interaction. The $4 p$ level Hamiltonian $H_{4 p}$ is decoupled from the other terms, and can be replaced with a constant $\varepsilon_{4 p}$ for the intermediate state, which we drop from $H_{\mathrm{el}}$. At a particular resonant $\omega_{\text {in }}$ for the $K$-edge RIXS, the matrix element of $H_{\mathrm{el}-\mathrm{pht}} \sim \mathbf{p} \cdot \mathbf{A}$ between $1 s$ and $4 p$ is a constant factor, leading to

$$
\begin{gathered}
I\left(\omega_{\text {in }}, \omega_{\text {out }}\right) \propto \sum_{f}\left|\sum_{n} \frac{\langle f|\underline{s}| n\rangle\left\langle n\left|\underline{s}^{\dagger}\right| i\right\rangle}{E_{i}+\omega_{\text {in }}-E_{n}+i \Gamma}\right|^{2} \\
\delta\left(E_{f}+\omega_{\text {out }}-E_{i}-\omega_{\text {in }}\right),
\end{gathered}
$$

where $\underline{s}^{\dagger}$ is a $1 s$ hole creation operator. Since transition metal $K$-edge RIXS probes initial and final states without core holes and intermediate states with core holes, $|i\rangle$ and $|f\rangle$ are the energy eigenstates of $H_{\mathrm{el}}$ with $\underline{s}^{\dagger} \underline{s}=0$, that is, $H_{3 d}$, whereas $|n\rangle$ are the energy eigenstates of $H_{\mathrm{el}}$ with $\underline{s}^{\dagger} \underline{s}=1$. Equation (5) is evaluated by expanding $3 d$ parts of $|n\rangle$ in the basis of the energy eigenstates of $H_{3 d}$, which include $3 d$ parts of $|i\rangle$ and $|f\rangle$.

In terms of a $3 d$ electron creation operator $d^{\dagger}$, the $3 d$ Hamiltonian for metals and insulators is

$$
H_{3 d}^{\mathrm{m} / \mathrm{i}}=\sum_{k} \varepsilon_{k} d_{k}^{\dagger} d_{k}
$$

for which we consider a constant DOS without and with a gap $(\Delta)$ for metals and insulators, respectively. The Hamiltonian includes $N$ one-electron $3 d$ levels. The number of $3 d$ electrons is $L$. For independent electrons with a single orbital degree of freedom at each site, the two spin channels are equivalent, and two bound states are present at the site with the core hole. However, for many transition-metal compounds, the Coulomb interaction between valence electrons is often non-negligible, resulting in the Mott insulators. For these Mott insulators, we consider the Hubbard Hamiltonian,

$$
H_{3 d}^{\mathrm{Hubbard}}=\sum_{k, \sigma=\uparrow, \downarrow} \varepsilon_{k} d_{k, \sigma}^{\dagger} d_{k, \sigma}+U \sum_{i} d_{i \uparrow}^{\dagger} d_{i \uparrow} d_{i \downarrow}^{\dagger} d_{i \downarrow},
$$

which includes the effect of the on-site $3 d-3 d$ Coulomb interaction. Spin and site indices are $\sigma$ and $i$, respectively.

Because the $1 s$ band is much narrower than the $3 d$ band, the total RIXS intensity is, in a good approximation, proportional to the intensity calculated for a $1 \mathrm{~s}$ hole created at, for example, site $i=0$ (see footnote 19). Therefore, we use $1 s$ hole Hamiltonian,

$$
H_{\underline{1 s}}=\varepsilon_{1 s} \underline{s}^{\dagger} \underline{s} .
$$

The following identity shows that the on-site Coulomb interaction with a localized core hole scatters any $3 d$ state with a wavevector $k$ into the same or any other $3 d$ state with a wavevector $k^{\prime}$ with an equal probability,

$$
-U_{c} d_{i=0}^{\dagger} d_{i=0} \underline{s}_{i=0}^{\dagger} \underline{s}_{i=0}=-\sum_{k, k^{\prime}} \frac{U_{c}}{N} d_{k}^{\dagger} d_{k^{\prime}} \underline{s}_{i=0}^{\dagger} \underline{s}_{i=0} .
$$

For our model, this corresponds to the scattering from any $3 d$ level into the same or any other $3 d$ level with the same strength of $-U_{c} / N$, which is expressed in the Hamiltonian,

$$
H_{\underline{1 s}-3 d}=-\sum_{k, k^{\prime}} \frac{U_{c}}{N} d_{k}^{\dagger} d_{k^{\prime}} \underline{s}^{\dagger} \underline{s} .
$$

Our model Hamiltonian for metals and insulators with the core hole, that is, $H_{\mathrm{el}}=H_{3 d}^{\mathrm{m} / \mathrm{i}}+H_{\underline{1 s}-3 d}+H_{\underline{1 s}}$ with $\underline{s}^{\dagger} \underline{s}=1$, is an exactly-solvable independent-particle Hamiltonian, and has been solved in the context of $\mathrm{x}$ ray edge singularity. ${ }^{20,21}$ The intermediate $L$-electron eigenstates and eigenenergies are obtained by diagonalizing $H_{\mathrm{el}}$ exactly and filling $L$ one-electron eigenstates. To calculate the RIXS intensity, we classify final states according to the number of e-h pairs, for example, the Fermi sea (i.e., the initial state $|i\rangle$ ), states with one e-h pair $d_{k_{>}}^{\dagger} d_{k_{<}}|i\rangle$, and states with two e-h pairs $d_{k_{>}}^{\dagger} d_{k_{>}^{\prime}}^{\dagger} d_{k_{<}} d_{k_{<}^{\prime}}|i\rangle$, where $k_{<}$and $k_{>}$represent $3 d$ electron levels below and above the Fermi energy, respectively. We examine the contribution of each class of states to the RIXS intensity, as we increase the number of e- $h$ pairs in the final states.

For the Mott insulators, the Hubbard model cannot be solved exactly. However, it is known that the main features of the Mott insulators are understood by following

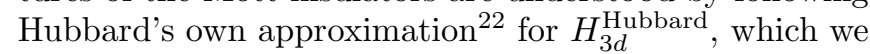
extend to calculate the RIXS spectral line shape for the half-filled Mott insulators. First, we project the operator for a particular spin onto different occupations of the opposite spin,

$$
d_{i \sigma}^{\dagger}=\left(1-n_{i,-\sigma}\right) d_{i \sigma}^{\dagger}+n_{i,-\sigma} d_{i \sigma}^{\dagger} .
$$


The first operator (singlon) $g_{i \sigma}^{\dagger}=\left(1-n_{i,-\sigma}\right) d_{i \sigma}^{\dagger}$ describes the motion of the electron in the absence of the electron with the opposite spin. The second operator (doublon) $b_{i \sigma}^{\dagger}=n_{i,-\sigma} d_{i \sigma}^{\dagger}$ describes the motion of the electron when the site is doubly occupied. The local potential in the presence of the core hole at $i=0$ is written as

$$
H_{\underline{1 s}-3 d}=-\sum_{\sigma} U_{c}\left(g_{i=0, \sigma}^{\dagger} g_{i=0, \sigma}+b_{i=0, \sigma}^{\dagger} b_{i=0, \sigma}\right) .
$$

In the Hubbard-I approximation, 22 the projection operators, $1-n_{i,-\sigma}$ and $n_{i,-\sigma}$, are replaced by their expectation values, resulting in an effective independent-particle Hamiltonian. The one-particle eigenvectors for the lower and upper Hubbard bands in the absence of the core-hole potential are written as

$$
\begin{aligned}
\left|\varepsilon_{\mathbf{k} L \sigma}\right\rangle & =\cos \theta_{\mathbf{k}}\left|g_{\mathbf{k} \sigma}\right\rangle+\sin \theta_{\mathbf{k}}\left|b_{\mathbf{k} \sigma}\right\rangle, \\
\left|\varepsilon_{\mathbf{k} U \sigma}\right\rangle & =\sin \theta_{\mathbf{k}}\left|g_{\mathbf{k} \sigma}\right\rangle-\cos \theta_{\mathbf{k}}\left|b_{\mathbf{k} \sigma}\right\rangle,
\end{aligned}
$$

respectively, where $\mathbf{k}$ is the wavevector. Although the core-hole potential in Eq. (12) does not couple singlon and doublon states, scattering between the lower and upper Hubbard bands still occurs due to the mixing of the singlon and doublon states through electron hopping. In the initial state, only the states in the lower Hubbard band are occupied,

$$
|i\rangle=\prod_{\mathbf{k} \sigma}\left|\varepsilon_{\mathbf{k} L \sigma}\right\rangle .
$$

For the intermediate states, the effective independentparticle Hamiltonian is solved in the presence of the corehole potential, leading to one-particle eigenstates $\left|\varepsilon_{m \sigma}\right\rangle$, where the index $m=1,2,3, \cdots$ labels the eigenstates in the order of increasing energy. We note that the wavevector $\mathbf{k}$ is no longer a good quantum number and that the lower and upper Hubbard bands are no longer well defined because of the presence of a bound state at the site with the core hole. Many-electron intermediate eigenstates and energies are obtained by filling an appropriate number of the states, $\left|\varepsilon_{m \sigma}\right\rangle$. By following the same procedure as for metals and insulators, we find the RIXS spectral shape for the Mott insulators.

\section{RESULTS}

To find RIXS spectrum, all possible intermediate states should be considered in principle, as done for small clusters in Refs. 13 and 14. However, for most RIXS experiments, the incoming x-ray energy is tuned at the absorption threshold for the resonance enhancement effect. Therefore, without the short lifetime of the intermediate state and the energy uncertainty, the intermediate state would be purely the lowest energy state, in which the localized core hole is screened by the $3 d$ electrons. To find the contribution of different intermediate states to the RIXS intensity, we calculate a part of the RIXS matrix

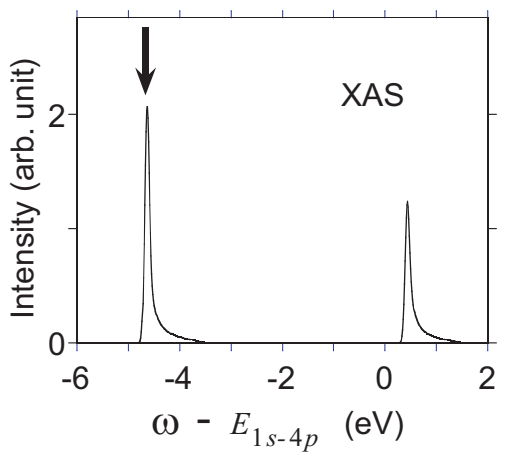

FIG. 2: X-ray absorption spectroscopy (XAS) spectrum for a metallic DOS with a bandwidth $W=2 \mathrm{eV}$ and a core-hole potential $U_{c}=5 \mathrm{eV}$ at half-filling.

element $\left\langle n\left|\underline{s}^{\dagger}\right| i\right\rangle$ in Eq.(5), namely the projection of the intermediate states onto the state of the Fermi sea with a core hole, which corresponds to the x-ray absorption intensity,

$$
I_{\mathrm{XAS}}(\omega) \propto \sum_{n}\left|\left\langle n\left|\underline{s}^{\dagger}\right| i\right\rangle\right|^{2} \delta\left(\omega-E_{n}+E_{i}\right) .
$$

The x-ray absorption intensity, calculated for a square density of states with a band width $W=2 \mathrm{eV}$ and $U_{c}=5$ $\mathrm{eV}$ at half-filling, is displayed in Fig. 2, which shows two distinct spectral bands corresponding to the XAS final states with an almost full (screened) and an almost empty (unscreened) bound state, separated by approximately $U_{c}$ (Ref. 20). Experimentally, the energy difference between the onsets of resonant inelastic x-ray scattering for a screened and an unscreened core hole is 5-7 eV (Ref. [5), which corresponds to $U_{c}$. For the lower spectral band, the XAS spectral weight is dominated by the transitions into the lowest energy intermediate state, $\left|n_{\text {low }}\right\rangle$, whereas the higher-lying states in this band have little component of the Fermi sea with a core hole, $\underline{s}^{\dagger}|i\rangle$. It shows that the lowest energy screening state, indicated by an arrow in Fig. 2 dominates the matrix element. The smaller satellite peak at higher energy corresponds to the excitation of the unscreened state. Since the core-hole lifetime broadening $\Gamma$ is significantly smaller than the energy difference between the screened and unscreened absorption states, the probability for the unscreened state excitation would be low. Even if the unscreened state is partially excited, its matrix element for RIXS is smaller than that for the screened state. Based on these arguments, we propose that the screened state is the dominant intermediate state contributing to the RIXS intensity, and we replace the sum over the intermediate states with a single lowest energy intermediate state, $n_{\text {low }}$ :

$$
I(\Delta \omega) \propto \sum_{f}\left|\left\langle f|\underline{s}| n_{\mathrm{low}}\right\rangle\right|^{2} \delta\left[\Delta \omega-\left(E_{f}-E_{i}\right)\right] .
$$

For metals and insulators, using indices $k$ and $m$, which are reserved for $3 d$-level one-electron eigenstates with- 
out and with the core hole respectively throughout this paper, we express operators for the intermediate oneparticle eigenstates with energy $\varepsilon_{m}(m=1,2, \ldots, N$ in the order of increasing energy) as

$$
D_{m}^{\dagger}=\sum_{k} a_{m k} d_{k}^{\dagger}
$$

The numerically exact lowest energy intermediate state $\left|n_{\text {low }}\right\rangle$ is found by filling up the lowest $L$ one-electron energy eigenstates of $H_{\mathrm{el}}$ with $\underline{s}^{\dagger} \underline{s}=1$.

The RIXS intensity in Eq. 17] calculated for the final states with up to three e-h pairs for a half-filled metallic DOS is shown by the upper (black) solid line marked with $\Delta=0$ in Fig. 3(a). The full bandwidth and the core-hole potential are chosen as $W=2 \mathrm{eV}$ and $U_{c}=5$ $\mathrm{eV}$, respectively. The RIXS intensity calculated only for the final states with two e-h pairs is shown by the lower (green) solid line marked with $\Delta=0$ in Fig. [3(a), which is small compared to the total intensity. The contribution of the final states with three e-h pairs to the RIXS intensity is negligible. The results show that the final states with one e-h pair contribute dominantly to the RIXS intensity, which means that the lowest intermediate state is approximately a combination of the Fermi sea and the states with one e-h pair,

$$
\left|n_{\mathrm{low}}\right\rangle \approx \alpha_{\mathrm{low}} \underline{s}^{\dagger}|i\rangle+\sum_{k_{>}, k_{<}} \beta_{\mathrm{low}}\left(k_{>}, k_{<}\right) d_{k_{>}}^{\dagger} d_{k_{<}} \underline{s}^{\dagger}|i\rangle .
$$

The first term gives rise to a large $\left\langle n_{\text {low }}\left|\underline{s}^{\dagger}\right| i\right\rangle$, the XAS part in the RIXS matrix element in Eq. (5) as discussed earlier. The remaining terms with one e-h pair give a large $\left\langle f|\underline{s}| n_{\text {low }}\right\rangle$, the other part in the RIXS matrix element.

The electron and the hole states in $\left|n_{\text {low }}\right\rangle$ are entangled in general. We examine whether unentangled electron and hole states serve as an approximation, that is,

$$
\begin{aligned}
& \left|n_{\text {low }}\right\rangle \approx \alpha_{\text {low }} \underline{s}^{\dagger}|i\rangle+ \\
& \left(\sum_{k_{>}} \beta_{\text {low }}^{e}\left(k_{>}\right) d_{k_{>}}^{\dagger}\right)\left(\sum_{k_{<}} \beta_{\text {low }}^{h}\left(k_{<}\right) d_{k_{<}}\right) \underline{s}^{\dagger}|i\rangle .
\end{aligned}
$$

We calculate the electron and hole distribution functions for the lowest energy intermediate state using $a_{m k}$ defined in Eq. (18),

$$
\begin{aligned}
& n_{k_{>}}^{e}\left(\varepsilon_{k_{>}}\right)=\sum_{m=1, L}\left|a_{m k_{>}}\right|^{2}, \\
& n_{k_{<}}^{h}\left(\varepsilon_{k_{<}}\right)=\sum_{m=L+1, N}\left|a_{m k_{<}}\right|^{2},
\end{aligned}
$$

which are shown in Fig. 3(b) for $\Delta=0$. If Eq. (20) is exact, we would have

$$
\begin{aligned}
& n_{k_{>}}^{e}\left(\varepsilon_{k_{>}}\right) \propto\left|\beta_{\text {low }}^{e}\left(k_{>}\right)\right|^{2}, \\
& n_{k_{<}}^{h}\left(\varepsilon_{k_{<}}\right) \propto\left|\beta_{\text {low }}^{h}\left(k_{<}\right)\right|^{2}
\end{aligned}
$$
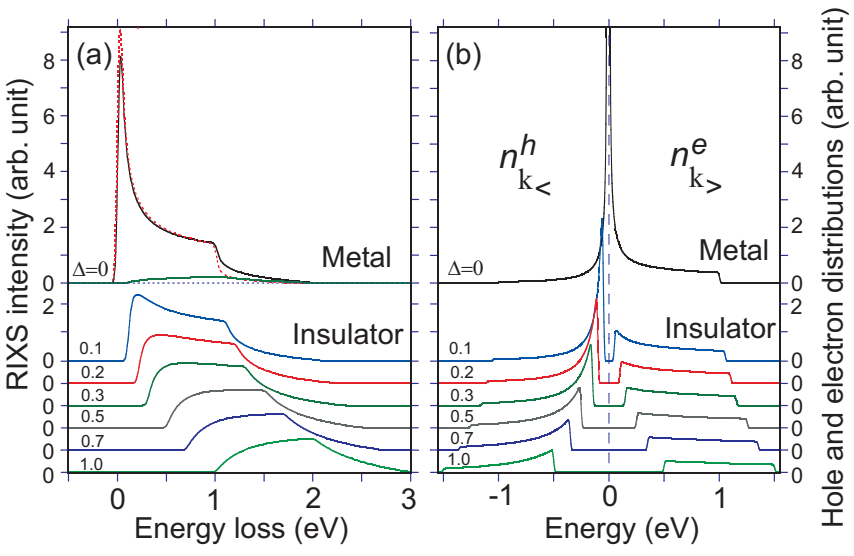

FIG. 3: (color online) (a) RIXS spectra calculated for a square density of states with a band width $W=2 \mathrm{eV}$ and $U_{c}=5$ $\mathrm{eV}$ at half-filling for a metal [upper (black) solid line for $\Delta=$ $0]$ and insulators $(\Delta>0)$. For the insulators, a gap $(\Delta)$ is opened at the center of the band, and the occupied and unoccupied bands have the same width $W / 2$. For the metal $(\Delta=0)$, the lower (green) solid line shows the contribution of the double electron-hole pairs to the RIXS spectrum and the upper (red) dotted line gives the approximate spectrum using Eq. (25). The lower (green) dotted line is a shifted energy axis. (b) Distribution functions of holes $\left(n_{k_{<}}^{h}\right)$ and electrons $\left(n_{k_{>}}^{e}\right)$ below and above the Fermi level for different values of $\Delta$, created by the intermediate-state core-hole potential.

and

$$
I(\Delta \omega)=A \sum_{k_{>}, k_{<}} n_{k_{>}}^{e}\left(\varepsilon_{k_{>}}\right) n_{k_{<}}^{h}\left(\varepsilon_{k_{<}}\right) \delta\left(\Delta \omega-\varepsilon_{k_{>}}+\varepsilon_{k_{<}}\right)
$$

Therefore, the comparison of the RIXS intensity calculated from Eq. (25) with the one calculated from Eq. (17) reveals whether Eq. (20) is a good approximation. The RIXS spectrum calculated from Eq. (25) is shown with the upper (red) dotted line for $\Delta=0$ in Fig. 3(a), which shows a good agreement with the upper (black) solid line, indicating that a separable e-h pair state is a good approximation for the lowest energy intermediate state.

We examine the characteristics of the electron and hole distribution functions, $n^{e}\left(\varepsilon_{k_{>}}\right)$and $n^{h}\left(\varepsilon_{k_{<}}\right)$, shown in Fig. 3(b) for a metal $(\Delta=0)$. First, the distribution functions show a strong singularity near the Fermi level, which is responsible for a similar singularity in the RIXS intensity, $I(\Delta \omega)$. These features correspond to the low energy e-h excitations right near the Fermi energy, related to the x-ray edge singularity. ${ }^{23}$ However, the elastic peak in RIXS overlaps with this edge singularity, and higher energy features are more important. Away from the Fermi energy, hole spectral weight, $n_{k_{<}}^{h}\left(\varepsilon_{k_{<}}\right)$, diminishes very rapidly, whereas the electron spectral weight, $n_{k_{>}}^{e}\left(\varepsilon_{k_{>}}\right)$, remains nearly constant. We interpret this difference in the following way. In the lowest energy intermediate state, the electron screens the localized core hole, meaning that the excited electron should be localized in the real space. That corresponds to the state with 
a nearly constant amplitude in $k$-space, or a constant amplitude in the energy space since we assume a constant electron DOS. The excited $3 d$ hole does not screen the core hole, but is in a delocalized state right below the Fermi energy to minimize its kinetic energy, which corresponds to the spectral weight concentrated near the Fermi energy. Such asymmetry gives rise to the higher energy features in RIXS. The RIXS intensity for $\Delta=0$ in Fig. 3 is large up to $1 \mathrm{eV}$, i.e., the width of the unoccupied band, beyond which the intensity quickly decreases to zero. This asymmetry differs significantly from the existing approaches in Refs. 3, 6 and 16, which predict that the RIXS spectral line shape is proportional to the dynamic structure factor $S(\omega)$ (i.e., the convolution of the occupied and the unoccupied DOS) weighted by the energy of the shake-up structure. According to these studies, the RIXS for a square DOS would be given by $S(\omega)$, which has a triangular shape, multiplied by a $U_{c}^{2} / \omega^{2}$ (Refs. 3 and 6 ) or a $U_{c}^{2} /\left(U_{c}-\omega\right)^{2}$ (Ref. 16) energy dependence. However, Fig. 3(a) clearly shows strong deviations from a simple triangular line shape. The results in Refs. 3 and 6 are correct in the limits of $U_{c} \rightarrow 0$ and $U_{c} \rightarrow \infty$, respectively. For intermediate $U_{c}$, the screening dynamics becomes more important, leading to the asymmetry in electron and hole excitations.

We also carry out similar calculations for insulators. The results are shown in Fig. 3 for $\Delta>0$. For a small gap, the singularity disappears and the RIXS spectral line shape resembles more closely the unoccupied DOS than $S(\omega)$. For larger gap values of the order of the bandwidth, the spectral line shape develops into a triangular shape. The electron-hole asymmetry between $n_{k_{<}}^{h}$ and $n_{k_{>}}^{e}$ becomes weaker, but persists. It shows that the widths of RIXS peaks for metals and insulators with gaps smaller than the band width roughly correspond to the widths of unoccupied bands. Therefore, RIXS probes mainly unoccupied bands, complementing ARPES, which probes occupied bands.

The RIXS for the half-filled Mott insulators is calculated by the same procedure used for metals and insulators. For the lowest intermediate state, the lowest $N$ one-particle eigenstates are filled,

$$
\left|n_{\text {low }}\right\rangle=\prod_{\sigma=\uparrow, \downarrow, m=1, \cdots, N / 2}\left|\varepsilon_{m \sigma}\right\rangle .
$$

De-excitations from the lowest intermediate state are obtained by representing $\left|\varepsilon_{m \sigma}\right\rangle$ in terms of $\left|\varepsilon_{\mathbf{k} L \sigma}\right\rangle$ and $\left|\varepsilon_{\mathbf{k} U \sigma}\right\rangle$, and calculating the overlap with final e-h pair excitations, such as

$$
|f\rangle=\left|\varepsilon_{\mathbf{k}_{>} U \sigma^{\prime}}\right\rangle \prod_{\mathbf{k} \sigma \neq \mathbf{k}_{<} \sigma^{\prime}}\left|\varepsilon_{\mathbf{k} L \sigma}\right\rangle .
$$

The RIXS spectrum is calculated according to Eq. (17). The results for $U=1,2$, and $3 \mathrm{eV}$ are shown in Fig. 4(a), where we consider final states with up to two e-h pairs. Electron and hole distribution functions for the Mott insulators in Fig. 4(b) show even stronger asymmetry than

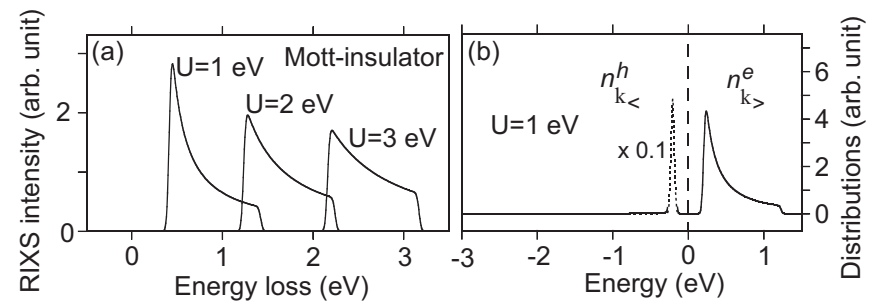

FIG. 4: (color online) (a) RIXS spectra calculated for the halffilled Mott insulators with the on-site Coulomb interaction $U=1,2$, and $3 \mathrm{eV}\left(U_{c}=5 \mathrm{eV}, W=2 \mathrm{eV}\right)$. (b) Distribution functions of holes and electrons created by the intermediate core-hole potential for the Mott insulator with $U=1 \mathrm{eV}$.

those for metals and insulators. The width of the RIXS peak is about $W / 2$, which is the width of the unoccupied (upper Hubbard) band. More discussions are given in the next section.

\section{DISCUSSIONS}

We analyze the relations between the one-particle eigenstates with and without the core hole to understand the results obtained in Sect. III. As mentioned in Sect. II, the Hamiltonians with the core-hole potential have been studied in the context of x-ray absorption edge singularity, $\stackrel{20,21}{2}$ and it is known that the coefficient relating the two basis sets in Eq. (18) is

$$
a_{m k}=\frac{C_{m}}{\varepsilon_{k}-\varepsilon_{m}},
$$

where $C_{m}$ is a normalization factor and $\varepsilon_{m}$ 's are the solutions of

$$
\frac{1}{U_{c}}=\frac{1}{N} \sum_{k} \frac{1}{\varepsilon_{k}-\varepsilon_{m}} .
$$

Figure 1 in Ref. 20 illustrates how $\varepsilon_{m}$ is determined graphically for a given set of $\varepsilon_{k}$ 's and a value of $U_{c}$.

First, we analyze the results for metals. For a metallic DOS with $N$ states separated by an equal, small energy difference, the effect of the strong attractive Coulomb potential $-U_{c}$ is to pull out a bound state below the bottom of the band and shift the energies of the remaining $N-1$ states (see Fig. 1 in Ref. 20). The calculated DOS in the presence of the core-hole potential is shown with the gray (green) solid line in Fig. 5(b). The bound state energy is $\varepsilon_{m=1} \cong-U_{c}-\frac{W^{2}}{12 U_{c}}$. The energies for the rest of the states are $\varepsilon_{m}=\varepsilon_{k}-\frac{\delta_{m}}{\pi} \frac{W}{N}$, where the indices $m$ and $k$ have the same value and the phase shift is $\delta_{m}=\pi, \ldots, 0$ for $\varepsilon_{k}=-\frac{W}{2}, \ldots, \frac{W}{2}$ in the large $U_{c}$ and $N$ limit. As indicated by Eqs. (21) and (22), $a_{m k_{>}}$'s with $m=1, \ldots, L$ correspond to electron excitations, because they represent levels unoccupied initially but occupied in the intermediate state. Similarly, 
$a_{m k_{<}}$'s with $m=L+1, \ldots, N$ correspond to hole excitations. The expression of $a_{m k}=C_{m} /\left(\varepsilon_{k}-\varepsilon_{m}\right)$ indicates that the squared amplitude of a state $k$ in a state $m$ decreases as their energy difference increases, proportional to $\left(\varepsilon_{k}-\varepsilon_{m}\right)^{-2}$. Therefore, if the intermediate one-electron energy $\varepsilon_{m}$ is well separated from a band of $\varepsilon_{k}$, like the bound state $m=1$, all $\varepsilon_{k}$ states in the band would contribute almost equally to that intermediate state. In contrast, if the intermediate state en$\operatorname{ergy} \varepsilon_{m}$ is right next to some $\varepsilon_{k}$ 's, like the intermediate states in the continuum band, those nearby $\varepsilon_{k}$ states contribute dominantly to that intermediate state. Therefore, as indicated by the long (blue) arrow between Figs. [5(a) and 5(b) and the dotted line in Fig. 5(a), the intermediate bound state includes electron excitations with an almost constant distribution function above the Fermi energy. The intermediate state in the continuum includes electron or hole excitation only if the intermediate state is right near the Fermi energy, as indicated by the two short (pale blue and red) arrows between Figs. 5(a) and 5(b), giving rise to singular features. This idea is further demonstrated by the (red) dashed line in Fig. 5 (b), which represents $n_{m}^{e}=\sum_{k>} a_{m k>}^{2}$ for $m=1, \ldots, L$, the levels with energies below zero, and $n_{m}^{h}=\sum_{k<} a_{m k<}^{2}$ for $m=L+1, \ldots, N$, the levels with energies above zero. The distribution functions $n_{m}^{e}$ and $n_{m}^{h}$ represent how different intermediate eigenstates contribute to the the e-h excitations, whereas $n_{k_{<}}^{h}$ and $n_{k_{>}}^{e}$ represent how different initial and final eigenstates contribute to the the e-h excitations.

We apply a similar analysis to insulators, as shown in Figs. 5(c) and 5(d). The intermediate state DOS is shown in gray (green) solid line in Fig. 5(d). It indicates two bound states, one below the bottom of the lower band and the other within the gap. Other $L-1$ and $N-L-1$ states are within the lower and upper continuum bands, respectively, with energies shifted in a similar way as metals. Therefore, the $L$ electrons fill right to the top of the lower band. In the limit of $U_{c}, \Delta \gg W$, the two bound state energies are $-\frac{U_{c}}{2} \pm \frac{1}{2} \sqrt{U_{c}^{2}+\Delta^{2}}$. Further approximation in the limit of $U_{c} \gg \Delta$ gives rise to the bound state energy below the bottom of the band, $\varepsilon_{m=1} \cong-U_{c}-\Delta^{2} /\left(4 U_{c}\right)$, and the bound state energy inside the gap, $\varepsilon_{m=L+1} \cong \Delta^{2} /\left(4 U_{c}\right)$. The (red) dashed line in Fig. [5(d) shows $n_{m}^{e}$ for $m=1, \ldots, L$ and $n_{m}^{h}$ for $m=L+1, \ldots, N$. Because of the gap, the intermediate continuum states do not include e-h excitation, and therefore, the singularity in $n_{k_{>}}^{e}$ or $n_{k_{<}}^{h}$ disappears. The states with substantial electron and hole excitations are the bound states, $\varepsilon_{m=1}$ and $\varepsilon_{m=L+1}$, respectively, as shown with the arrows between Figs. 5 (c) and 5 (d). The shape of electron and hole distribution functions, $n_{k_{>}}^{e}$ and $n_{k_{<}}^{h}$, are determined by the distance between the intermediate bound states and the initially occupied or unoccupied bands. The bound state below the lower band is well separated from the initially unoccupied band, giving rise to an electron distribution function close to a constant. In contrast, the bound state within the gap

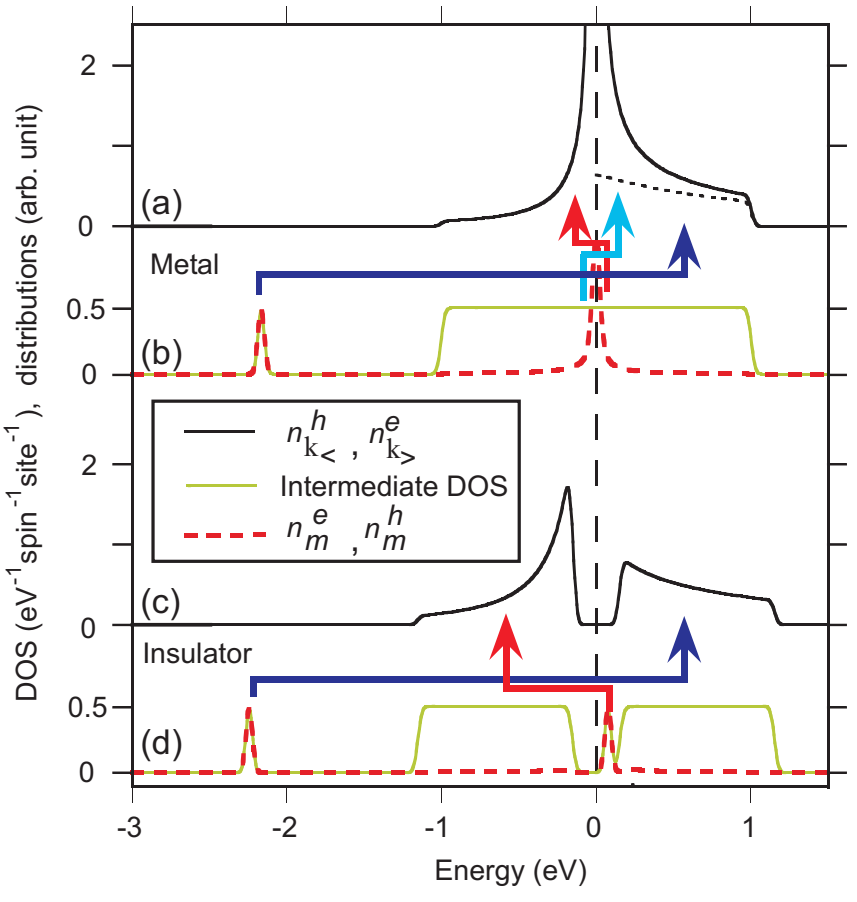

FIG. 5: (Color online) (a) and (c) show the distribution functions of holes and electrons in the intermediate state for a metal and an insulator $(\Delta=0.3 \mathrm{eV})$ respectively, similar to the ones shown in Fig. 3(b). (b) and (d) show the intermediate DOS with gray (green) solid lines and $n_{m}^{e}$ and $n_{m}^{h}$, defined in the text, with (red) dashed lines. Arrows indicate how the two plots are related, as explained in the text. For illustrative purpose, a smaller core-hole potential $\left(U_{c}=2 \mathrm{eV}\right)$ is chosen. Other parameter values are identical to those for Fig. 3.

is close to the top of the initially occupied band, which gives rise to a hole distribution function peaked near the gap. This difference results in the strong asymmetry in the electron and hole distributions.

The results for the half-filled Mott insulators shown in Fig. 4 are interpreted in a similar way. The on-site Coulomb interaction splits the DOS into the lower and upper Hubbard bands (LHB and UHB) with a gap proportional to $U$. With two spin degrees of freedom on the site with the core hole, two bound states are pulled below the bottom of the LHB, as shown with the (green) solid line in Fig. 6(b). The lowest bound state at approximately $U_{c}$ below the LHB consists mainly of the states from the LHB. The second bound state, which is about $U$ higher in energy, is made of mainly the states from the UHB. For the lowest intermediate state, both bound states are filled, giving an energy of $-2 U_{c}+U$, which is equivalent to the energy for two electrons at the site with a core hole. After the de-excitation, these bound electrons scatter to different states. However, the LHB is already occupied in the ground state and the lowest bound state does not contribute to the inelastic scattering, as indicated by $n_{m}^{e}$ and $n_{m}^{h}$ shown in the (red) dashed line in Fig. 6(b). In contrast, very few doubly occupied 


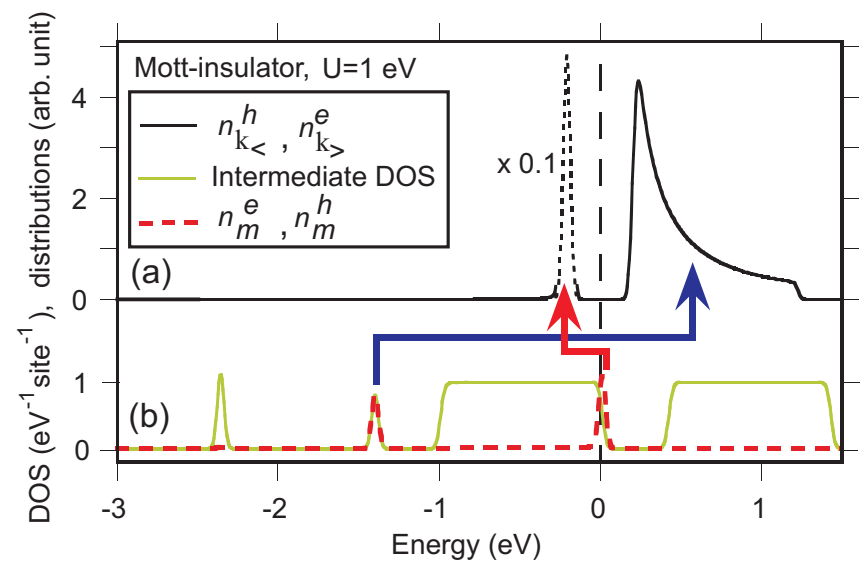

FIG. 6: (color online) (a) Occupation of holes and electrons for the Mott insulator with $U=1 \mathrm{eV}$, similar to the one shown in Fig. 4(b). For illustrative purpose, a smaller corehole potential $\left(U_{c}=2 \mathrm{eV}\right)$ is chosen. (b) Intermediate DOS shown in gray (green) solid line and $n_{m}^{e}$ and $n_{m}^{h}$, defined in the text, in (red) dashed line. Arrows indicate how the two plots are related, as explained in the text.

states are present in the ground state and the second bound state leads to the scattering to all the states in the UHB. After filling the two bound states, $N-2$ electrons are left to fill $N-1$ states in the LHB in the intermediate state. Therefore, the lowest intermediate state has a hole at the top of the LHB, and the hole scatters only to a narrow region of the occupied DOS, as shown in Figs. 6)(a) and 6(b). The RIXS spectral line shape therefore mainly reflects the unoccupied DOS, as is clear from the RIXS spectra in Fig. 4(a).

The relationship between the RIXS spectrum and the dynamic structure factor is one of the interests in recent studies. Our results on the forms of $n_{k_{<}}^{h}$ and $n_{k_{>}}^{e}$ for metals and insulators allow us to find explicit expressions of $I(\omega)$ in certain limits, which can be compared with the dynamic structure factor $S(\omega)$ directly. For example, in the limit of $U_{c} \gg \Delta, W$, we approximate $n_{k_{>}}^{e} \sim U_{c}^{-2}$ for $\frac{\Delta}{2} \leq \varepsilon_{k_{>}} \leq \frac{\Delta+W}{2}$, and $n_{k_{<}}^{h} \sim\left(\varepsilon_{k_{<}}\right)^{-2}$ for $-\frac{\Delta+W}{2} \leq$ $\varepsilon_{k_{<}} \leq-\frac{\Delta}{2}$. From these and Eq. (25), we find the RIXS spectrum and its relation to the dynamic structure factor. For $\Delta \leq \omega<\Delta+\frac{W}{2}$, we obtain

$$
I(\omega) \sim S(\omega) /\left[\frac{\Delta}{2}\left(\omega-\frac{\Delta}{2}\right)\right],
$$

where $S(\omega) \sim \omega-\Delta$. For $\Delta+\frac{W}{2} \leq \omega<\Delta+W$,

$$
I(\omega) \sim S(\omega) /\left[\frac{W+\Delta}{2}\left(\omega-\frac{W+\Delta}{2}\right)\right],
$$

where $S(\omega) \sim \Delta+W-\omega$. This shows that the relation between RIXS intensity and the dynamic structure factor is not as straight-forward as expected from previous studies. As for the integrated spectral weight, we find that it approaches to $A L(N-L) / N^{2}$ in the limit of $U_{c} \gg \Delta \gg W$, recovering the results found in Ref. 16 .

Materials with narrow unoccupied and wide occupied bands provide testing grounds for our results, since our theory predicts a narrow RIXS spectrum with the hole excitations close to the Fermi level, whereas a simple convolution of the unoccupied and occupied states gives a wide RIXS spectrum. Recent calculations ${ }^{24}$ suggest that the undoped manganite $\mathrm{LaMnO}_{3}$ has such asymmetric band widths (about $0.4 \mathrm{eV}$ and $2 \mathrm{eV}$ for the unoccupied and the occupied bands, respectively) due to the layered $A$-type orbital ordering. The RIXS spectrum measured for this material24 indeed shows a narrow peak with a small dispersion (less than $0.5 \mathrm{eV}$ ), consistent with our theory. It would be also worthwhile to examine the RIXS spectrum for materials with prominent features in the occupied bands far below the Fermi level, which would distinguish our theory from a simple convolution. We further note that, when $\omega_{\text {in }}$ is tuned to the satellite (unscreened) peak in the XAS spectrum, the situation is reversed: a hole is bound to the core hole and an electron is excited near the Fermi energy. Such RIXS tuned at the satellite peak would probe occupied bands, instead of empty bands.

\section{SUMMARY}

In summary, we have shown that the spectral weight of charge excitations in transition metal $K$-edge RIXS, where the core-hole potential is larger than the effective bandwidth, is not determined by the total energy of the shake-up, but results mainly from the nature of the intermediate-state core-hole valence-shell-electron excitons. We have identified the strong difference between excited electrons and holes, which can be used to study occupied and unoccupied bands separately. The methodology described in this work can be extended to more complicated systems, for example, by incorporating results from $a b$ initio calculations, allowing a better interpretation of transition metal $K$-edge RIXS.

\section{Acknowledgments}

We thank John Hill, Stephane Grenier, and Jeroen van den Brink for discussions. Work at Argonne National Laboratory was supported by the U.S. Department of Energy, Office of Basic Energy Sciences, under contract W-31-109-Eng-38. MvV was supported by the U.S. Department of Energy (DE-FG02-03ER46097) and the Institute for Nanoscience, Engineering, and Technology under a grant from the U.S. Department of Education. 
1 C.-C. Kao, W. A. L. Caliebe, J. B. Hastings, and J.-M. Gillet, Phys. Rev. B 54, 16361 (1996).

2 J. P. Hill, C.-C. Kao, W. A. L. Caliebe, M. Matsubara, A. Kotani, J. L. Peng, and R. L. Greene, Phys. Rev. Lett. 80, 4967 (1998).

3 P. Abbamonte, C. A. Burns, E. D. Isaacs, P. M. Platzman, L. L. Miller, S. W. Cheong, and M. V. Klein, Phys. Rev. Lett. 83, 860 (1999).

${ }^{4}$ M. Z. Hasan, E. D. Isaacs, Z.-X. Shen, L. L. Miller, K. Tsutsui, T. Tohyama, and S. Maekawa, Science 288, 1811 (2000); M. Z. Hasan, P. A. Montano, E. D. Isaacs, Z.-X. Shen, H. Eisaki, S. K. Sinha, Z. Islam, N. Motoyama, and S. Uchida, Phys. Rev. Lett. 88, 177403 (2002).

5 Y. J. Kim, J. P. Hill, C. A. Burns, S. Wakimoto, R. J. Birgeneau, D. Casa, T. Gog, and C. T. Venkataraman, Phys. Rev. Lett. 89, 177003 (2002); Y.-J. Kim, J. P. Hill, H. Benthien, F. H. L. Essler, E. Jeckelmann, H. S. Choi, T. W. Noh, N. Motoyama, K. M. Kojima, S. Uchida, D. Casa, and T. Gog, ibid. 92, 137402 (2004).

${ }^{6}$ G. Döring, C. Sternemann, A. Kaprolat, A. Mattila, K. Hämäläinen, and W. Schülke, Phys. Rev. B 70, 085115 (2004).

7 J. P. Hill, G. Blumberg, Y.-J. Kim, D. Ellis, S. Wakimoto, R. J. Birgeneau, S. Komiya, Y. Ando, B. Liang, R. L. Greene, D. Casa, and T. Gog, arXiv:0709.3274 (unpublished); F. Forte, L. J. P. Ament, and J. van den Brink, Phys. Rev. B 77, 134428 (2008).

8 E. Saitoh, S. Okamoto, K. T. Takahashi, K. Tobe, K. Yamamoto, T. Kimura, S. Ishihara, S. Maekawa, Y. Tokura, Nature 410, 180 (2001).

9 H. Kondo, S. Ishihara, and S. Maekawa, Phys. Rev. B 64, 014414 (2001).

10 S. Grenier, J. P. Hill, V. Kiryukhin, W. Ku, Y.-J. Kim, K. J. Thomas, S-W. Cheong, Y. Tokura, Y. Tomioka, D. Casa, and T. Gog, Phys. Rev. Lett. 94, 047203 (2005).

11 F. Gel'mukhanov and H. Ågren Phys. Rev. B 57, 2780 (1998).

12 M. van Veenendaal and P. Carra, Phys. Rev. Lett. 78, 2839 (1997).

13 K. Tsutsui, T. Tohyama, and S. Maekawa, Phys. Rev. Lett. 83, 3705 (1999); ibid. 91, 117001 (2003).
14 T. Nomura and J.-I. Igarashi, J. Phys. Soc. Jpn. 73, 1677 (2004); T. Nomura and J.-I. Igarashi, Phys. Rev. B 71, 035110 (2005).

15 F. Vernay, B. Moritz, I. S. Elfimov, J. Geck, D. Hawthorn, T. P. Devereaux, and G. A. Sawatzky, Phys. Rev. B 77, 104519 (2008).

16 J. van den Brink and M. van Veenendaal, J. Phys. Chem. Solids, 66, 2145 (2005); Europhys. Lett., 21121 (2006); L. J. P. Ament, F. Forte, and J. van den Brink, Phys. Rev. B 75, 115118 (2007).

17 T. Ide and A. Kotani, J. Phys. Soc. Jpn. 68, 3100 (1999).

18 G. van der Laan, C. Westra, C. Haas, and G. A. Sawatzky, Phys. Rev. B 23, 4369 (1981); P. J. W. Weijs, M. T. Czyzyk, J. F. van Acker, W. Speier, J. B. Goedkoop, H. van Leuken, H. J. M. Hendrix, R. A. de Groot, G. van der Laan, K. H. J. Buschow, G. Wiech, and J. C. Fuggle, ibid. 41, 11899(1990).

19 Even for a dispersionless core-hole band, the momentum $\vec{k}$ is still a good quantum number because of the translational symmetry of the system. The core hole is directly coupled to the valence shell. If we create a valence shell excitation with a momentum $\vec{q}$, the core hole shifts also by a momentum $\vec{q}$. In the end, the momentum transferred to the valence shell equals the momentum lost by the photon. Therefore, in principle, we can calculate the momentum dependence by splitting the processes according to the momentum $\vec{q}$ of the excited e-h pairs. The $K$-edge RIXS measures the integrated spectrum, which is calculated in this paper.

20 L. C. Davis and L. A. Feldkamp, J. Appl. Phys. 50, 1944 (1979).

21 L. A. Feldkamp and L. C. Davis, Phys. Rev. B 22, 4994 (1980).

22 J. Hubbard, Proc. Roy. Soc. London Ser. A 276238 (1963).

23 G. D. Mahan, Phys. Rev. 163, 612 (1967); P. Nozières and C. T. De Dominicis, ibid. 178, 1097 (1969).

24 T. Inami, T. Fukuda, and J. Mizuki, S. Ishihara, H. Kondo, H. Nakao, T. Matsumura, K. Hirota, Y. Murakami, S. Maekawa, and Y. Endoh, Phys. Rev. B 67, 045108 (2003). 